\title{
Impact of haptic feedback on applied intracorporeal forces using a novel surgical robotic system-a randomized cross-over study with novices in an experimental setup
}

\author{
Johanna Miller ${ }^{1} \cdot$ Manuel Braun ${ }^{2} \cdot$ Johannes Bilz $^{3} \cdot$ Sebastian Matich ${ }^{3} \cdot$ Carsten Neupert $^{3} \cdot$ Wolfgang Kunert $^{1}($ ) \\ Andreas Kirschniak ${ }^{1}$
}

Received: 23 April 2020 / Accepted: 10 July 2020 / Published online: 22 July 2020

(c) The Author(s) 2020

\begin{abstract}
Background Most currently used surgical robots have no force feedback; the next generation displays forces visually. A novel single-port robotic surgical system called FLEXMIN has been developed. Through an outer diameter of $38 \mathrm{~mm}$, two instruments are teleoperated from a surgeon's control console including true haptic force feedback. One additional channel incorporates a telescope, another is free for special instrument functions.

Methods This randomized cross-over study analyzed the effect of haptic feedback on the application of intracorporeal forces. In a standardized experiment setup, the subjects had to draw circles with the surgical robot as gently as possible. The applied forces, the required time spans, and predefined error rates were measured.

Results Without haptic feedback, the maximum forces (median/IQR) were $6.43 \mathrm{~N} / 2.96 \mathrm{~N}$. With haptic feedback, the maximum forces were lower $(3.57 \mathrm{~N} / 1.94 \mathrm{~N}, p<0.001)$. Also, the arithmetic means of the force progression $(p<0.001)$ and their standard deviations $(p<0.001)$ were lower. Not significant were the shorter durations and lower error rates. No sequence effect of force or duration was detected. No characteristic learning or fatigue curve was observed.

Conclusions In the experiment setup, the true haptic force feedback can reduce the applied intracorporeal robotic force to one-half when considering the aspects maximum, means, and standard deviation. Other test tasks are needed to validate the influence of force feedback on surgical efficiency and safety.
\end{abstract}

Keywords Surgical robot $\cdot$ Haptics $\cdot$ Force feedback $\cdot$ Randomized controlled trial $\cdot$ Experiment setup

\section{Regaining the sense of touch}

In a surgical environment, haptics plays an important role for tissue assessment and interaction. Surgeons mainly use tactile and force feedback to identify anatomical structures

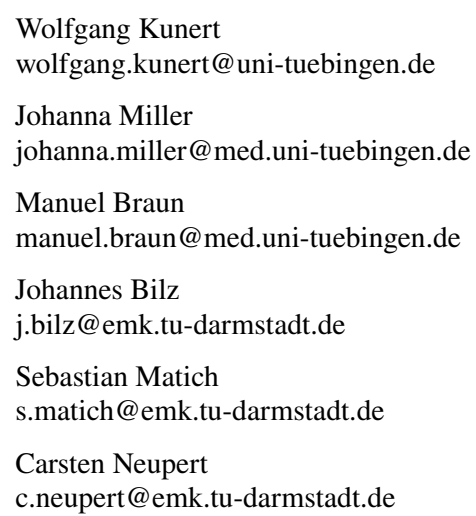

like vessels or nerves, to distinguish between healthy and diseased tissue as well as for instrument control. The role of haptic feedback in surgery is still widely discussed, especially since the introduction of robotically assisted procedures [1]. When using a master-slave setup, the

\author{
Andreas Kirschniak \\ andreas.kirschniak@uni-tuebingen.de \\ 1 Clinic of General, Visceral and Transplant Surgery, Working \\ Group Surgical Technology and Training, Tübingen \\ University Hospital, Waldhörnlestrasse 22, 72072 Tübingen, \\ Germany \\ 2 Clinic for Orthopaedics, Tübingen University Hospital, \\ Waldhörnlestrasse 22, 72072 Tübingen, Germany \\ 3 Department of Electromechanical Design, Darmstadt \\ Technical University, Merckstrasse 25, 64283 Darmstadt, \\ Germany
}


instrument-tissue interaction point and the surgeon's hand are physically disconnected. As a result, all conceivable haptic feedback is completely eliminated [2, 3]. Restoring the haptic information involves a three-step procedure: tactile or force assessment, digital signal transformation, and display as a perceivable feedback in the haptic interface. In general surgery, no generic haptic feedback is in clinical use to date. However, technical realization has already been proven in experimental setups [4, 5]. Possible causes are high demands made of the sensors such as sterilizability, insensitivity to influences within the abdominal cavity, and also the imitation of true haptic perception.

\section{"True haptic perception"}

With this term we describe force feedback without changing the somatosensory modality. The opposite could be pseudo-haptic feedback, where visual and tactile vibrations are transmitted to symbolize grasping forces between the instrument jaws [6, 7]. To differentiate the sensor channels, we take a closer look at the tactile sense when describing a passive recognizing process of several different tactile stimuli. It includes static proprioception (spatial awareness), visceroception (visceral signaling), nociception (perception of pain), thermoception (perception of temperature differences), and surface sensitivity (perception of mechanical irritations such as compression, vibration,, and elongation). Some tactile sensations are perceived with direct conjunction to kinaesthetic receptors [8]. The combination of passive tactile feedback (tissue perception) and dynamic kinaesthetic feedback (motion perception, as part of proprioception) is considered haptic feedback $[9,10]$. Consequently, haptics generally stands for an active procedure of exploration and requires interaction with an object. It can be synonymously described as a "tentative understanding." For the interpretation of all sensory information,, we make use of different exploratory procedures: unsupported holding, enclosure, applied pressure, lateral motion,, and contour following [11-13]. The pursued object properties to be determined by a surgeon are mainly size, surface, and material characteristics as well as position.

\section{Sensitive endoscopic surgeons}

Minimally invasive surgeons usually draw conclusions regarding tissue texture by visually examining the tissue surface. However, with the exclusive use of visual feedback, the subsurface stays hidden. For detailed tissue assessment, visual feedback has to be combined with kinaesthetic feedback in the course of dynamic examination. Tissue elasticity, for instance, can be estimated by observing the tissue deformation during force application [14]. Some studies have shown that providing both visual and kinaesthetic feedback is superior to solely visual or kinaesthetic feedback in terms of tissue characterization $[15,16]$. Other working groups have argued that direct or indirect visual monitoring during tasks performed minimally invasively can completely compensate the absence of haptic feedback [17]. It is undeniable that additional visual information definitely leads to an improvement in a person's motor skills [18]. However, the reliance on exclusively visual feedback attempts to make a virtue out of a necessity since no haptic feedback is available to date. The effect of reduced haptic feedback is also seen in laparoscopic surgery. Boer et. al [19] found that sensitivity feedback qualities of commercially available laparoscopic dissectors are eight times inferior to those of bare fingers. Besides the elimination of direct contact with the object (no tactile feedback), the transformation of applied forces through the instrument shaft is of special interest (modified kinaesthetic feedback) [3, 20, 21]. Force quality is influenced by inverted movement directions (fulcrum effect). Force quantity is affected by torsion forces in the abdominal wall and friction forces in the trocar [22].

\section{Experiment haptic setup}

To investigate the role of haptic feedback in robotic surgery, a novel telerobotic master-slave operating system, called FLEXMIN (Fig. 1) [23, 24], was developed in a cooperation between Tübingen University Hospital and Darmstadt Technical University. The aim and result of this R\&D project funded by the German Research Foundation (DFG) was a single-port system that, in particular, can be inserted rectally as for transanal endoscopic micro-surgery (TEM/TEO [25]). It incorporates two instruments remote-controlled from a surgeon's control console with force feedback. The slave robot with an outer diameter of $38 \mathrm{~mm}$ has a camera channel for a long telescope and a working channel for insertion of special assistance instruments (Fig. 1C).

\section{Research question}

We still lack evidence whether haptic feedback gives additional information to the surgeon, which then helps improve surgical techniques and reduce perioperative complications. Consequently, the relevance of haptics in this context remains unclear [18]. The aim of the present study is to quantify the impact of tactile perception on applied forces using the surgical robot FLEXMIN in an experiment setup. 


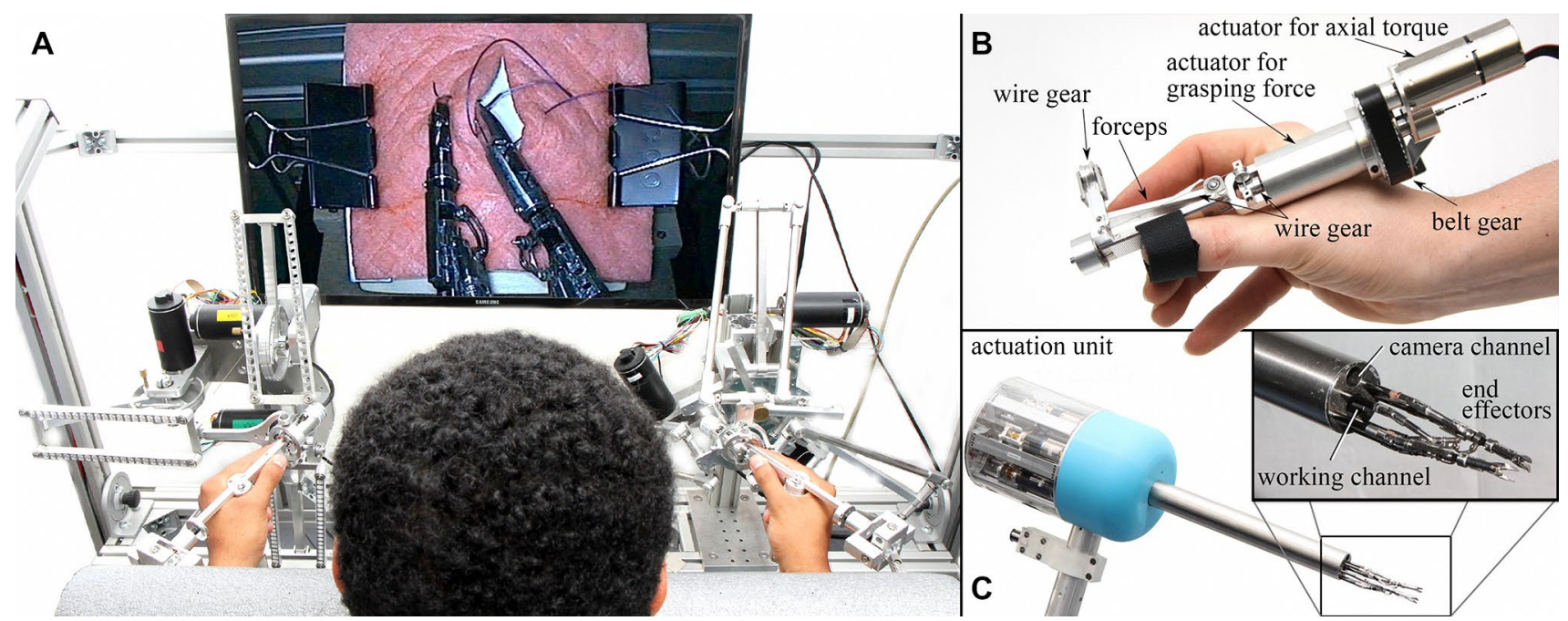

Fig. 1 Single-port master-slave robotic system FLEXMIN. A Surgeon's control console with haptic interface. B Haptic handle with force feedback. C Intracorporeal single-port instrument set with activation unit

\section{Materials and methods}

\section{Master-slave system FLEXMIN}

The presented study was conducted with the FLEXMIN system (Fig. 1), a single-port surgical robot (outer shaft diameter $38 \mathrm{~mm}$, two instruments with four degrees of freedom, Simulink real-time target machine, sampling rate $4 \mathrm{kHz}$, hardware connected via EtherCAT bus, bandwidth of force sensor $\sim 6 \mathrm{kHz}$, and delay between force measurement and feedback ( $1 \mathrm{~ms})$. The study subjects were operated the right FLEXMIN slave instrument with their right hand at the right haptic interface (Fig. 1B) [26, 27]. FLEXMIN's control unit recorded the trajectories of the instrument tip and also its applied force with the help of an additional 6-axis force/ torque (f/t) sensor (Nano17-E, ATI Industrial Automation, Apex, NC, USA). For live image presentation, a laparoscope (R. Wolf, $10 \mathrm{~mm}, 25^{\circ}$ viewing direction, two-dimensional) and an endocam (R. Wolf, 3CCD, PAL) were used. The subjects read their task instructions on an LCD monitor on their right side and viewed the endovideo on a second LCD monitor (24") in front of them.

\section{Study group}

The study group included 31 subjects with no experience in laparoscopic or robotically assisted surgical interventions. Exclusion criteria were any movement disorder or other neurological disease conditions influencing the motor system of the upper body. Necessary precondition for inclusion was at least average motor coordination skills when completing the Purdue Pegboard Test [28]. Three subjects were left-handed, but not excluded because they scored sufficient results on the
Purdue Pegboard Test using their non-dominant right hand. One student was not able to complete the test phase due to technical failure. All participants granted their written consent before joining the study. After enrollment, the subjects were randomly and equally allotted to two groups (Fig. 4).

\section{Standardized test task}

The test task called for precise positioning and moving of the right instrument tip. Paper sheets were vertically positioned in front of the master-slave console in systematic consideration of ergonomic posture. To ensure standardized conditions, each test round was conducted on a new paper sheet. For warm-up, a task with four equal crosses and straight dotted lines was used (training task, Fig. 2). The main task showed three circles of various size and drawn with a dotted line (test task, Fig. 3). Installed behind the sheets was the

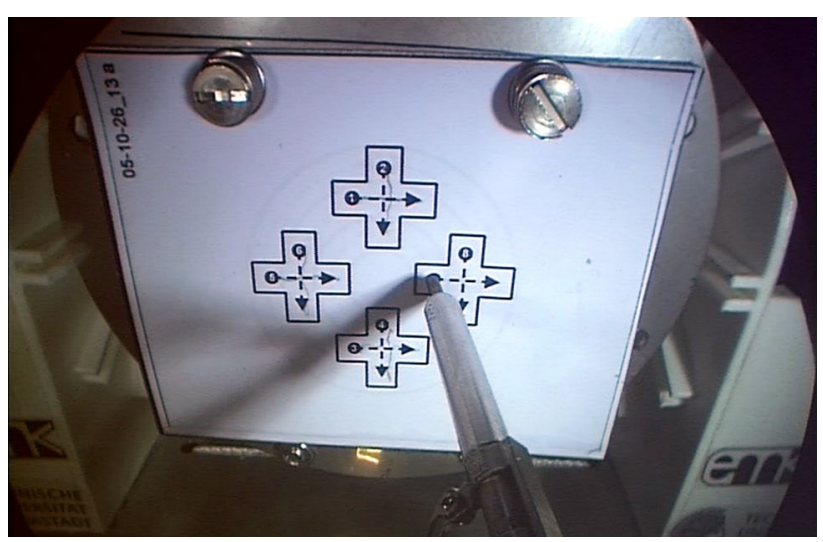

Fig. 2 Training task 


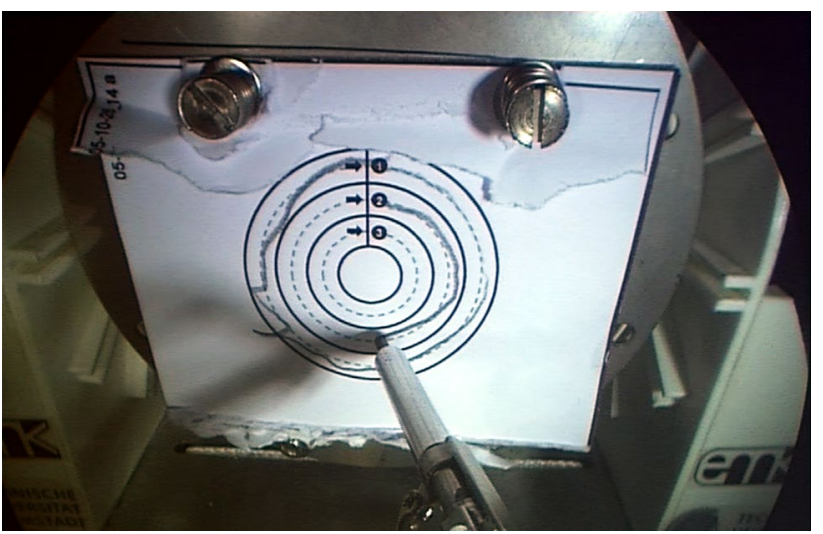

Fig. 3 Test task

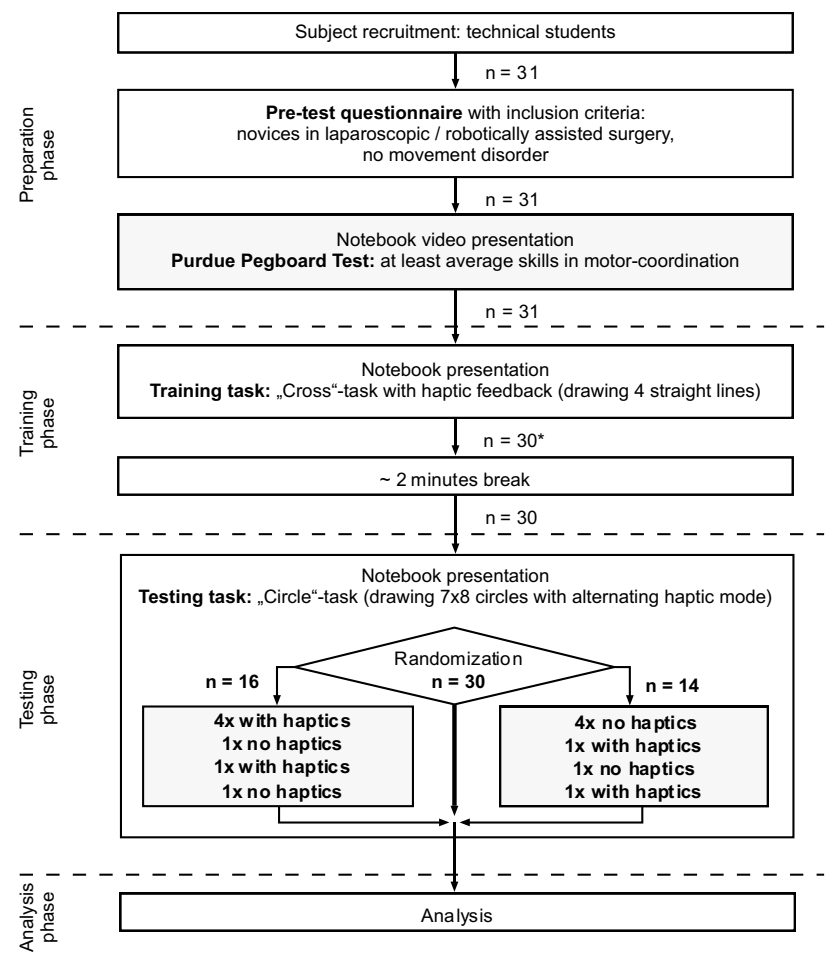

Fig. 4 Study design. *The last subject \#31 could not finish the training phase because the robotic system experienced a technical problem

force/torque sensor for measurement of the applied touching force in XYZ direction. The aim was to continuously trace the dotted straight and concentric lines within predetermined limits, with minimal force and without lifting the pencil lead. The pencil lead was fixed to the right robot effector. Leaving the boundaries was counted as an X/Y error. Lifting the pencil lead off the paper sheet was counted as a $\mathrm{Z}$ error. The reason for choosing a planar circle to be drawn as the test task was to avoid several kinds of bias. Firstly, the task should be as easy as possible to understand in order to avoid information bias. Secondly, all subjects know how to drawn a circle on a flat paper. Therefore, there is no learning curve for the manual action itself. Thirdly, following the circular curve located in space requires continuous readjustment of movement in all three directions in space. Fourthly, grasping actions would involve an additional grasping force that would overlay the drawing force. Since both have to be transmitted with the same handle, movement and grasping forces might interfere with each other. The drawing action avoids such a bias. The drawing of crosses as a warm-up task was designed to be even more simple, while also requiring continuous gentle contact with the paper.

\section{Statistical endpoints}

Primary statistical endpoint was the assessment of the applied maximum touching force. Our hypothesis was that applied forces are reduced when using haptic feedback. The time required for performance of each circle and the number of $\mathrm{X} / \mathrm{Y}$ and $\mathrm{Z}$ errors were defined as second endpoints.

\section{Study design}

The presented study was a two-armed prospective trial including 31 participants. The number of participants was identified by considering effect sizes. The diagram (Fig. 4) shows the study design as described below. All subjects completed a pre-test questionnaire asking for individual parameters such as age, sex, and handedness. Three subjects were left-handed and 28 were right-handed. With regard to time management and technical realization, students had to complete an abbreviated version of the Purdue Pegboard Test as instructed by a video and while referring to the Purdue Pegboard Test Manual. All instructions were shown as text on an additional monitor to the right of the endo monitor. The two tasks were demonstrated in brief videos shown on the same monitor. The first task was about picking up and placing items with the right hand (three times, $30 \mathrm{~s}$ each time). The second task was a bimanual assembly test procedure (three times, one minute each time). All 31 study participants showed at least average manual skills. For the purpose of familiarizing themselves with the master-slave console, a training phase followed. The training task instructions were demonstrated in a notebook presentation. The subjects were encouraged to trace four crosses consisting of eight straight dotted lines within given borders by operating the master-slave console with activated haptic feedback. Because of a technical failure on the first test day, that day's last student was not able to complete the training task and was therefore excluded. After a 2-min break, the participants started the test phase. Under consideration of possible interindividual performance differences, a cross-over design was chosen for this phase. After another notebook presentation 
giving instructions for the test task, subjects were randomly divided into Group A $(n=16)$ and Group B $(n=14)$. The unequal group sizes were caused by the randomization combined with the unexpected end of the training phase. The test task was to trace three circular lines six times within predetermined limits (large external circle first, medium circle next, small inner circle last). Group A was started by performing four test repetitions with activated haptic feedback $(+)$, followed by one round without $(-)$ and the last round with haptic feedback $(+)$. Group B had the opposite sequence $(----+-)$. Consequently, a total number of $30 \times 6 \times 3=540$ circles were traced.

\section{Data acquisition and documentation}

The applied touching forces were recorded with Matlab software (The MathWorks, Inc., Natick, MA, United States) using the $\mathrm{f} / \mathrm{t}$ sensor. After the test task, $\mathrm{X} / \mathrm{Y}$ errors in the form of drifting lines were counted by two independent experts. $Z$ errors were recorded as force values of zero newtons and separately counted. Start and stop times for each circle were manually marked by pressing a digital switch and were also recorded with Matlab software. For additional documentation, performance of all test tasks was videoed and transferred to a PC/notebook via USB video grabber.

\section{Statistical analysis}

For statistical analysis, SPSS version 25 (IBM, Armonk, NY, United States) software was used. The groups were analyzed for normal distribution using the Shapiro-Wilk W test. For normally distributed measured values, the arithmetic means and standard deviations were calculated. For distributionfree data, the median values and the interquartile range (IQR) were calculated. No carryover effect caused by rising repetition number was found. If the measuring data (forces and performance times) were not normally distributed, the Wilcoxon test was used to analyze the significance. Probability values of $p<0.05$ were considered significant.

\section{Results}

\section{Demographics of participants}

Questions 1 to 3 of the pre-test questionnaire were asked for demographic details on the participants. The age of the 31 study participants ranged from 22 to 32 years $(25.1 \pm 2.2$ years); 29 were males and two were female. Twenty-eight subjects were right-handed, and three were left-handed.

Mean body weight was $78.7 \mathrm{~kg} \pm 16.1 \mathrm{~kg}$, and mean height was $181 \mathrm{~cm} \pm 7.9 \mathrm{~cm}$.
The questionnaire was also asked for regular manual activities. Of all study participants, $38.7 \%$ play an instrument (9.7\% keyboard instrument, 29\% string instrument), $64.5 \%$ do handicrafts (such as painting, drawing or sculpting) in their leisure time, $38.7 \%$ regularly play skill games and $77.4 \%$ play computer games $(51.6 \%$ more often than once a month, $25.8 \%$ even more often than once a week).

The parameters age, body weight, and height did not influence performance quality regarding force and time effort, either with or without haptic feedback.

\section{Perdue Pegboard test}

The results of the first Pegboard task (positioning with the right hand) do not allow any conclusions to be drawn regarding force or time expended by the subjects in the test phase, either with or without haptic feedback. The same applies for the bimanual assembly test. The maximum touching force (Fig. 5) and the total performance time (Fig. 6) depending on the geometric mean Pegboard score are illustrated below.

\section{First endpoint (touching force)}

All measured data were distribution-free. Without haptic feedback, the maximum forces (median/min.-max./IQR) were $6.43 \mathrm{~N} / 2.94 \mathrm{~N}-14.58 \mathrm{~N} / 2.964 \mathrm{~N}$. With haptic feedback, the maximum forces were significantly $(p<0.001)$ lower: $3.57 \mathrm{~N} / 1.30 \mathrm{~N}-10.23 \mathrm{~N} / 1.936 \mathrm{~N}$ (Fig. 7). When using haptic feedback, the mean forces also were significantly lower $(p<0.001) 1.72 \mathrm{~N} / 0.64 \mathrm{~N}-6.29 \mathrm{~N} / 1.42 \mathrm{~N}$ (Fig. 8), as well as the standard deviations of the force progressions $(p<0.001)$ $0.79 \mathrm{~N} / 0.23 \mathrm{~N}-2.05 \mathrm{~N} / 0.44 \mathrm{~N}$ (Fig. 9). No sequence effect was detected in the force (Fig. 10). No characteristic learning or fatigue curve was visible.

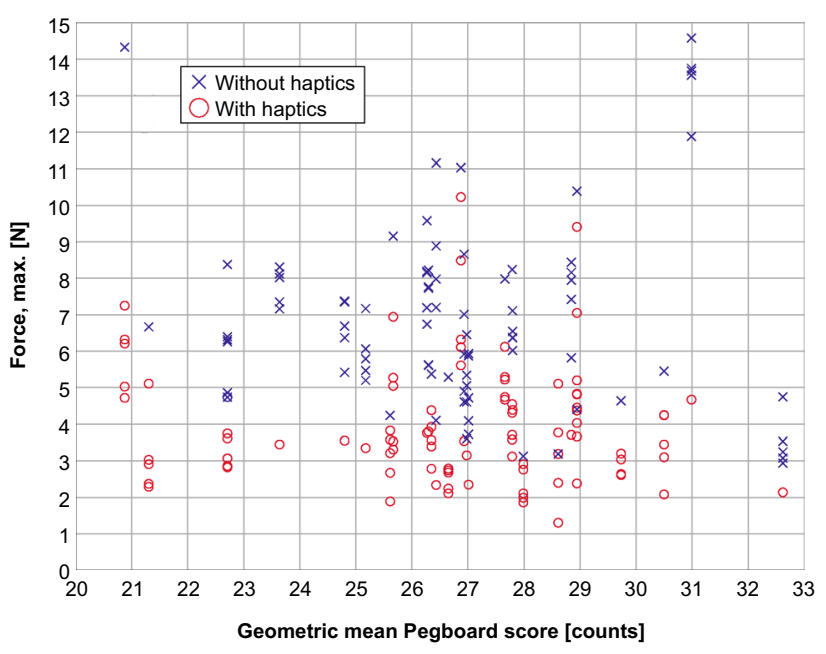

Fig. 5 Applied maximum force $[\mathrm{N}]$ depending on manual dexterity represented by the Perdue Pegboard Test score [counts] 


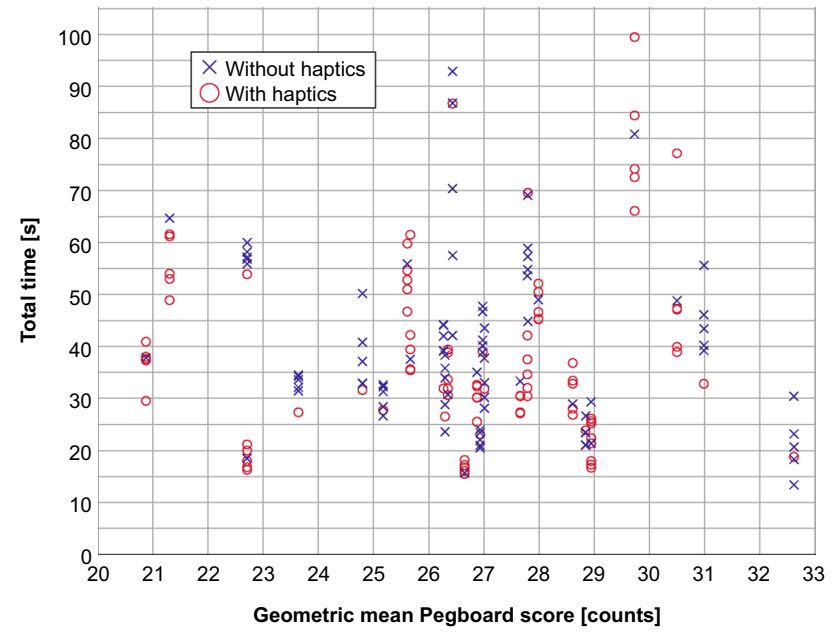

Fig. 6 Total performance time [s] depending on Perdue Pegboard Test score [counts]

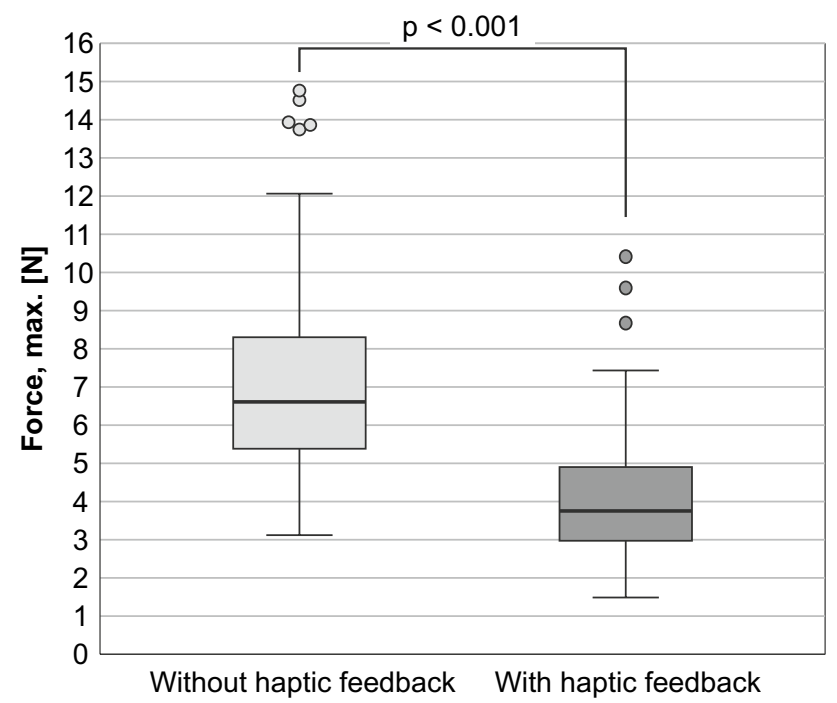

Fig. 7 Maximum applied force $[\mathrm{N}]$ depending on haptic mode [n=30]

\section{Second endpoints}

\section{$\mathrm{XY}$ and $\mathrm{Z}$ errors}

Similar to the first endpoint, no normal distribution was found for the second endpoints. The XY error rate (median/min.-max./IQR) without haptic feedback was $0 / 0-13.33 \% / 3.33 \%$. With haptic feedback, only the interquartile range differed and amounted to $0 \%$. The $\mathrm{Z}$ error rate without haptics was $6.67 / 0-93.33 \% / 16.67 \%$ versus $0 / 0-76.6 \% / 7 \%$ with haptics. Analysis of both parameters showed no statistical significance (Fig. 11).

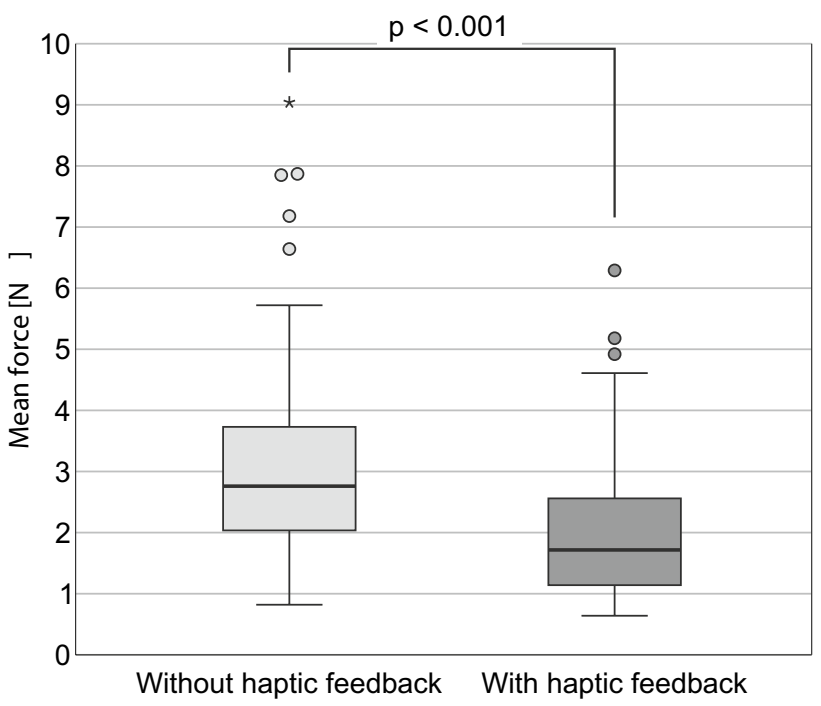

Fig. 8 Mean applied force $[\mathrm{N}]$ depending on haptic mode $[n=30]$

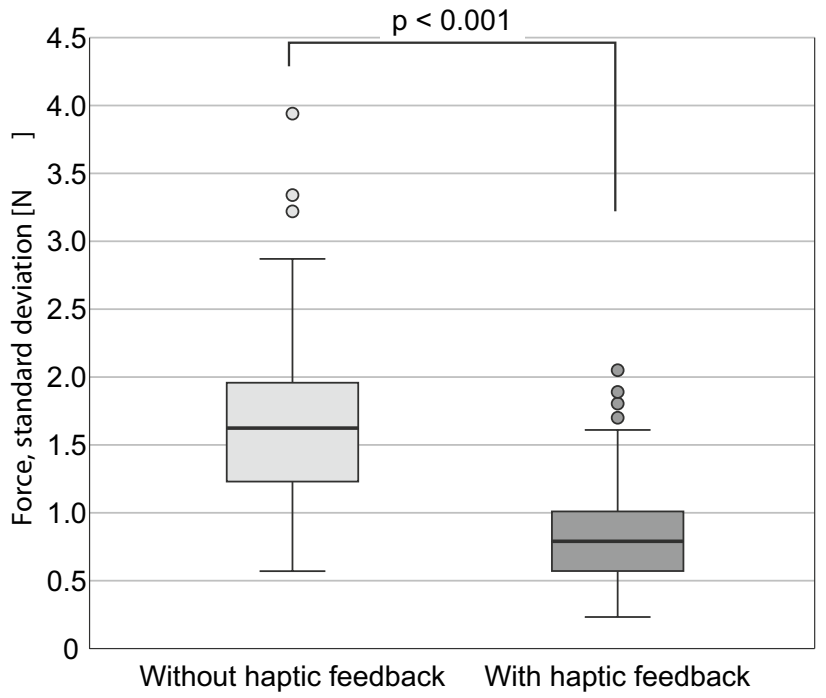

Fig. 9 Standard deviation of applied forces $[\mathrm{N}]$ depending on haptic mode $[n=30]$

\section{Performance time}

Median performance time was minimally lower in the haptic group (32.8 s versus $37.3 \mathrm{~s}$ in the group without haptics), but without significance (Fig. 12). For the minimal and maximum performance time as well as the interquartile range, haptic feedback showed no positive influence. 


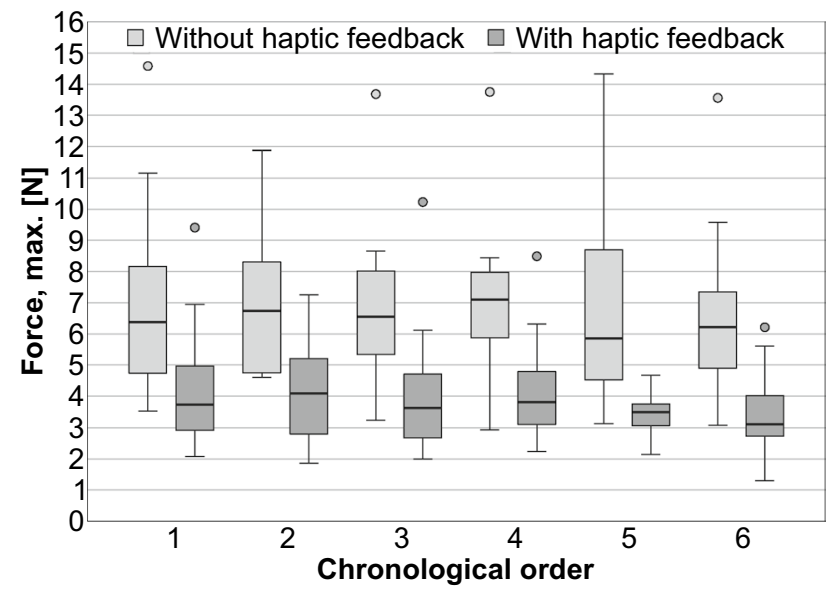

Fig. 10 Maximum applied force $[\mathrm{N}]$ depending on haptic mode and chronological order $[n=30]$

\section{Discussion}

The presented randomized comparative experiment trial showed a benefit of true haptic force feedback as indicated by a significant reduction in applied forces. In previous studies, maximum pulling forces were measured with the gall bladder in a box trainer [29]. The used traumatic graspers usually damaged the tissue when the pulling force exceeded a value of $23.3 \mathrm{~N} \pm 8.2 \mathrm{~N}$. In laparoscopy , such brute forces are applied only with large graspers, namely to tissue that will be extracted. When using atraumatic graspers, the tissue slipped out at pulling forces in a range of $11.5 \mathrm{~N} \pm 3.2 \mathrm{~N}$, whereby it is not clear how "atraumatic" these manipulations really were. In the present study, maximum forces ranged from 2.9 to $14.6 \mathrm{~N}$ without haptics and from 1.30 to $10.23 \mathrm{~N}$ with haptics. Consequently, this reduction of the applied intracorporeal robotic forces by one-half could mean the difference whether a tissue is damaged or not. For
Fig. 11 Error number depending on haptic mode $[n=30]$

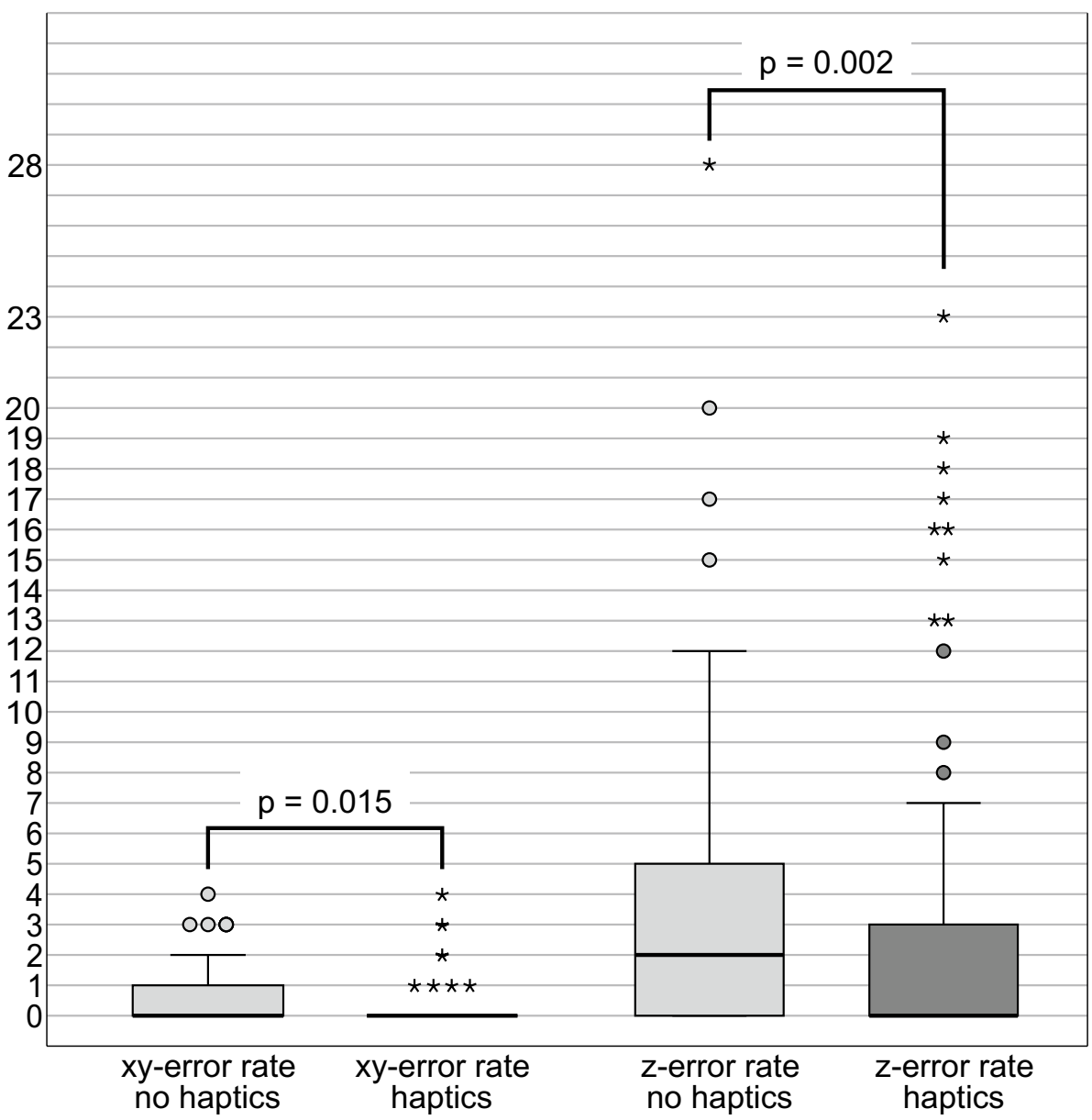




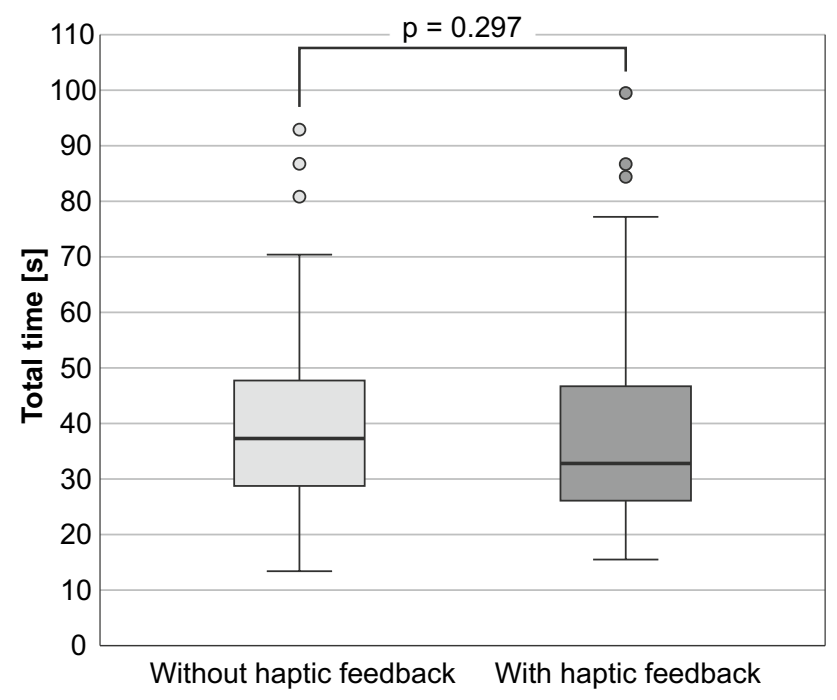

Fig. 12 Total performance time [s] depending on haptic mode $[n=30]$

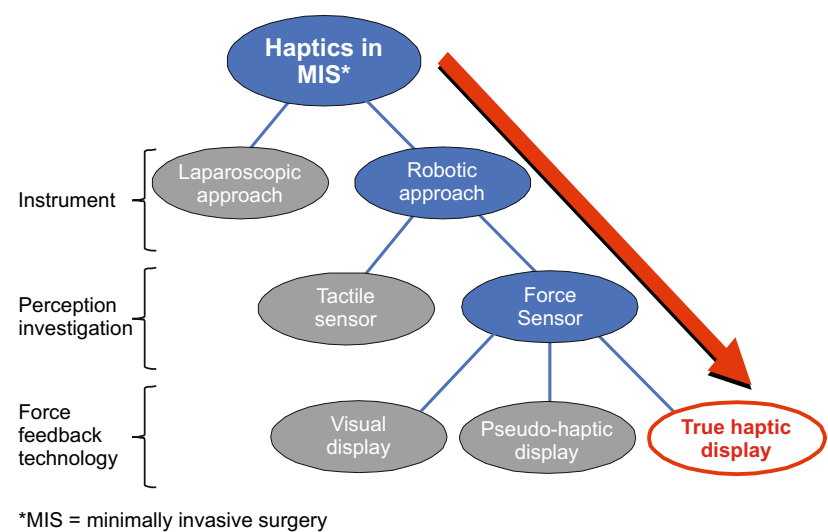

Fig. 13 Classification of surgical haptics in the literature

better interpretation of our results, we classified the complex topic of haptics (Fig. 13) reported in the literature. Firstly, our study analyzed haptics in a robotic not involved in the laparoscopic surgical approach. Secondly, we measured only the three-dimensional application force at the tip of the surgical instrument, but not the tactile surface information. Therefore, we delivered force/kinesthetic feedback and not tactile/cutaneous feedback. Thirdly, the force feedback was conveyed as a true haptic force feedback unlike visual or pseudo-haptic representation [30].

Visual feedback can contain a lot of information, but is principally not transparent due to the implied change in sensation channel. There is a consensus concerning the fact that especially novices perform significantly faster and with fewer errors in box trainers or simulator studies when using three-dimensional (3D) laparoscopic imaging systems [31].
If we had used 3D vision in our study, like in the da Vinci robotic system, the non-haptics group might have benefitted more than the 2D group. However, the pencil lead cast a shadow on the paper, thus clearly indicating the moment of touch. In addition, the notepad was slightly elastic. Hence, applying a touch force resulted in visible movement of the paper surface. On the other hand, 3D vision requires adaption and selection of the subjects. For this reason, the authors preferred to use two-dimensional vision with helpful illumination instead of 3D. Most existing haptic displays emerged from virtual reality environments and generate only a pseudo-haptic feedback presented by vibrations or fluid surface variations. Only a few cases of true haptic force feedback are reported $[32,33]$. However, until now, these concepts have not provided any proof of concept in general surgery. In future, haptic gloves might pose a new research area [34]. So far, also no surgical instrument has been clinically established that would allow imitation of the complex tactile movements between the fingertips as applied in open surgical procedures. Consequently, the only available tactile information is the three-dimensional instrument-tissue interaction force and possibly the grasping force [5]. This is why there is only little literature addressing surgical palpation with tactile sensors. Schostek et al. [35] technically described a tactile sensor with $3 \times 10$ tactile elements inside the instrument jaws. Perri et al. [36] evaluated a laparoscopic tactile sensing system with $4 \times 15$ tactile elements grasping ex vivo bovine liver tissue. The force distribution was shown visually in the form of a color contour pressure map and the total force in a bar chart. Perri found a $71 \%$ reduction in the applied maximum pressure as compared to an endoscopic grasper. However, his results are not comparable with ours, because the force was assessed between the jaws and not as a forward-pushing force exerted by the instrument tip. In addition, the feedback was displayed visually. Reiley et al. [37] hypothesized that visualization of applied forces is a haptic feedback surrogate. In his study, four surgeons with da Vinci experience and six novices completed a standardized knot tying task under different sensory substitution (no feedback, visual feedback, auditory feedback, combined audio-visual feedback). The applied force parameters were recorded by an instrument tracking system that was integrated in the da Vinci robot. The authors found that any sensory substitution scenario, alone or in combination, led to a measurable reduction of applied forces. However, the visual force feedback showed an advantage only for surgeons with no da Vinci training. The authors suspected that experienced surgeons are probably more adept at using visual cues for task completion [31]. Meccariello et al. [17] share the opinion that surgical experience can compensate the lack of haptic feedback. In an experimental palpation task, three membranes each with a different thickness had to be touched and then ordered according to hardness. In addition, a hidden metallic 
clip behind the membranes had to be identified. The expert surgeons scored 8.87 and significantly $(p<0.05)$ better than did the non-experts with a score of 3.57. They also logged a shorter performance time of $28.8 \mathrm{~s}$ compared to $71.3 \mathrm{~s}$. Since only novices participated in our study, it can be assumed that if experts had participated we might have found less benefit from the haptic feedback, because the experts would have found more visual cues for force control. However, the "expert effect" can not be carried over to our study, because the tasks and the scoring were quite different [32]. Haouchine et al. [38] presented an approach to indirect estimation of instrument-tissue interaction forces without any mechanical force sensor. A stereoscopic camera gathered two slightly different perspectives that were used to calculate a threedimensional biomechanical model on-the-fly. The interaction force was estimated from the computed tissue deformations and was visualized as an augmented live endovideo. Nevertheless, the system requires detailed surface textures and, difficult to access, subsurface elastic tissue properties. Sutherland et al. [32] designed an image-guided robot arm, called "neuroArm," that allows the visual presentation of positional and instrument-tissue force information. It was used to resect glioma in 18 patients. The elaborate system is specialized for neurosurgery offering micro-manipulation, high sensitivity, and MR compatibility. The two-armed robot is designed for use in an open operative site and supported by additional manual instruments. Nevertheless, even though the robot is not suitable for the laparoscopic approach, the haptic console is of special interest. The applied forces were about 10 times smaller than those applied in our laparoscopic scenario. By the way, the "neuroArm" robot and also a force-sensing surgical tool [39] use the same kind of force sensor that we applied in our study. In contrast to the aforementioned works, studies have shown that the absence of haptic feedback can lead to excessive and inadequate force application [26]. In this context, a larger number of incidents of tissue damage [40] or inappropriate suture handling [4] have been described. In our comparative study, task performance was subjected to neither a learning nor a fatigue curve. It can be assumed that the test task was brief enough to not impair the subjects' attention level. The highly significant results relativize a possible bias caused by the fact that the number of subjects in the two study arms differed slightly. Moreover, it seems that the handling of the FLEXMIN system was intuitive enough to perform the task straight away without a learning effect bias. This is the advantage of the standardized experimental setup. We are aware that it remains to be proven whether and how the impact of real haptic feedback can be transferred to clinical routine. So far, the FLEXMIN robotic system is a scientific tool and not approved for clinical use.

\section{Conclusion}

In the experiment setup, the true haptic force feedback can reduce the applied intracorporeal robotic force by one-half considering the aspects maximum, mean, and standard deviation. To ensure smooth operation with insensitive surgical robots, the surgeon must have learned to interpret visual cues of force application. This is possible for experienced robotic surgeons as opposed to the novices in our study, but is certainly not intuitive. It is desirable to restore force sensation for the robotically operating physician. Giving the robotic surgeon force feedback is sensible in any case, especially since haptics is natural and perfectly compatible with diligent visual observation. Further studies with different test tasks and subjects having varying surgical experience are needed to validate the influence of force feedback and pseudo-haptic feedback on surgical efficiency and safety.

Acknowledgements Open Access funding provided by Projekt DEAL. The FLEXMIN surgical robots project was funded by the German Research Foundation (DFG) under the reference numbers SCHL 532/62, WE 2308/13-2, KI1645/1-2.

\section{Compliance with ethical standards}

Disclosures J. Miller, J. Bilz, W. Kunert, Drs. M. Braun, S. Matich, C. Neupert, and Prof. A. Kirschniak have no conflicts of interests or financial ties to disclose.

Open Access This article is licensed under a Creative Commons Attribution 4.0 International License, which permits use, sharing, adaptation, distribution and reproduction in any medium or format, as long as you give appropriate credit to the original author(s) and the source, provide a link to the Creative Commons licence, and indicate if changes were made. The images or other third party material in this article are included in the article's Creative Commons licence, unless indicated otherwise in a credit line to the material. If material is not included in the article's Creative Commons licence and your intended use is not permitted by statutory regulation or exceeds the permitted use, you will need to obtain permission directly from the copyright holder. To view a copy of this licence, visit http://creativecommons.org/licenses/by/4.0/.

\section{References}

1. Wagner CR, Stylopoulos N, Howe RD (2002) The role of force feedback in surgery: analysis of blunt dissection. 10th Symposium on haptic interfaces for virtual environment and teleoperator systems, Proceedings. pp 73-79

2. Verner LN, Okamura AM (2009) Force \& torque feedback vs force only feedback. World haptics 2009: Third Joint Eurohaptics Conference and Symposium on haptic interfaces for virtual environment and teleoperator systems, Proceedings. pp 406-410

3. Okamura AM (2009) Haptic feedback in robot-assisted minimally invasive surgery. Curr Opin Urol 19:102-107 
4. Moody L, Baber C, Arvanitis TN (2002) Objective surgical performance evaluation based on haptic feedback. Stud Health Technol Inform 85:304-310

5. Paydar OH, Wottawa CR, Fan RE, Dutson EP, Grundfest WS, Culjat MO, Candler RN (2012) Fabrication of a thin-film capacitive force sensor array for tactile feedback in robotic surgery. 2012 Annual International Conference of the IEEE Engineering in Medicine and Biology Society. IEEE, Piscataway, pp 2355-2358

6. Wojcik SA, Neupert C, Bilz J, Werthschützky R, Kupnik M, Hatzfeld C (2018) Pseudohaptic feedback for teleoperated gripping interactions. In: Prattichizzo D, Ruffaldi E (eds) Lecture notes in computer science. Springer, Pisa, pp 309-320

7. Hachisu T, Cirio G, Marchal M, Lecuyer A, Kajimoto H (2011) Pseudo-haptic feedback augmented with visual and tactile vibrations. IEEE, Piscataway

8. Kálmán M, Csillag A (2005) The skin and other diffuse sensory systems. In: Csillag A (ed) Atlas of the sensory organs: functional and clinical anatomy. Humana Press, Totowa, pp 199-243

9. Loomis JM (1982) Analysis of tactile and visual confusion matrices. Percept Psychophys 31:41-52

10. Schostek S, Schurr MO, Buess GF (2009) Review on aspects of artificial tactile feedback in laparoscopic surgery. Med Eng Phys 31:887-898

11. Lederman SJ, Klatzky RL (1993) Extracting object properties through haptic exploration. Acta Psychol 84:29-40

12. Lederman SJ, Klatzky RL, Reed CL (1993) Constraints on haptic integration of spatially shared object dimensions. Perception 22:723-743

13. Lederman SJ, Reed CL (1993) Orthogonal redundant information about 3-D objects - constraints on haptic dimensional integration (Vol 30, Pg 446, 1992). B Psychonomic Soc 31:85-85

14. Enayati N, De Momi E, Ferrigno G (2016) Haptics in robot-assisted surgery: challenges and benefits. IEEE Rev Biomed Eng 9:49-65

15. Tavakoli M, Aziminejad A, Patel RV, Moallem M (2006) Methods and mechanisms for contact feedback in a robot-assisted minimally invasive environment. Surg Endosc Other Interv Tech 20:1570-1579

16. Tavakoli M, Patel RV, Moallem M (2006) Bilateral control of a teleoperator for soft tissue palpation: design and experiments. 2006 IEEE International Conference on Robotics and Automation (ICRA). IEEE, Piscataway, pp 3280-3285

17. Meccariello G, Faedi F, AlGhamdi S, Montevecchi F, Firinu E, Zanotti C, Cavaliere D, Gunelli R, Taurchini M, Amadori A, Vicini C (2016) An experimental study about haptic feedback in robotic surgery: may visual feedback substitute tactile feedback? J Robot Surg 10:57-61

18. van der Meijden OAJ, Schijven MP (2009) The value of haptic feedback in conventional and robot-assisted minimal invasive surgery and virtual reality training: a current review. Surg Endosc 23:1180-1190

19. den Boer KT, Herder JL, Sjoerdsma W, Meijer DW, Gouma DJ, Stassen HG (1999) Sensitivity of laparoscopic dissectors. What can you feel? Surg Endosc 13:869-873

20. Pinzon D, Byrns S, Zheng B (2016) Prevailing trends in haptic feedback simulation for minimally invasive surgery. Surg Innov 23:415-421

21. Bholat OS, Haluck RS, Murray WB, Gorman PJ, Krummel TM (1999) Tactile feedback is present during minimally invasive surgery. J Am Coll Surg 189:349-355

22. van den Dobbelsteen JJ, Schooleman A, Dankelman J (2007) Friction dynamics of trocars. Surg Endosc 21:1338-1343

23. Matich S, Neupert C, Schlaak HF, Pott PP (2016) A single port robot with parallel manipulators, latest results. 50th DGBMT Annual Conference, Basel

24. Hatzfeld C, Neupert C, Matich S, Braun M, Bilz J, Johannink J, Miller J, Pott PP, Schlaak HF, Kupnik M, Werthschutzky R, Kirschniak A (2017) A teleoperated platform for transanal single-port surgery: ergonomics and workspace aspects. 2017 IEEE World Haptics Conference (WHC). IEEE, Piscataway, pp 1-6

25. Sagae VMT, Ribeiro IB, de Moura DTH, Brunaldi VO, Logiudice FP, Funari MP, Baba ER, Bernardo WM, de Moura EGH (2020) Endoscopic submucosal dissection versus transanal endoscopic surgery for the treatment of early rectal tumor: a systematic review and meta-analysis. Surg Endosc 34:1025-1034

26. Neupert C, Klug F, Matich S, Kirschniak A, Pott PP, Schlaak HF, Werthschutzky R (2013) New device for ergonomic control of a surgical robot with 4 DOF including haptic feedback. Biomed Tech, Berlin

27. Thiem DB, Neupert C, Bilz J, Matich S, Polzin J, Werthschutzky R, Kupnik M, Schlaak HF, Kirschniak A, Hessinger M, Hatzfeld C (2017) User-interface for teleoperation with mixed-signal haptic feedback. IEEE International Conference on Intelligent Robots and Systems. IEEE, Vancouver, BC, pp 892-898

28. Buddenberg LA, Davis C (2000) Test-retest reliability of the Purdue Pegboard Test. Am J Occup Ther 54:555-558

29. Haas P, Kunert W, Johannink J, Kirschniak A (2017) Objective measurement of instrument-tissue interaction in laparoscopic surgery. In: Dössel O (ed) 51. Jahrestagung der Biomedizinischen Technik und Dreiländertagung der Medizinischen Physik. Walter de Gruyter, Berlin, Boston, Dresden

30. Neupert C, Matich S, Scherping N, Kupnik M, Werthschutzky R, Hatzfeld C (2016) Pseudo-haptic feedback in teleoperation. IEEE Trans Haptics 9:397-408

31. Arezzo A, Vettoretto N, Francis NK, Bonino MA, Curtis NJ, Amparore D, Arolfo S, Barberio M, Boni L, Brodie R, Bouvy N, Cassinotti E, Carus T, Checcucci E, Custers P, Diana M, Jansen M, Jaspers J, Marom G, Momose K, Muller-Stich BP, Nakajima K, Nickel F, Perretta S, Porpiglia F, Sanchez-Margallo F, SanchezMargallo JA, Schijven M, Silecchia G, Passera R, Mintz Y (2019) The use of 3D laparoscopic imaging systems in surgery: EAES consensus development conference 2018. Surg Endosc Other Interv Tech 33:3251-3274

32. Sutherland GR, Maddahi Y, Gan LS, Lama S, Zareinia K (2015) Robotics in the neurosurgical treatment of glioma. Surg Neurol Int 6:S1-S8

33. Brodie A, Vasdev N (2018) The future of robotic surgery-how robotics could help shape the future of surgical care. Ann Royal Coll Surg Engl 100:4-13

34. Park KC (2019) Haptic gloves and surgical robot systems. Google Patents

35. Schostek S, Zimmermann M, Schurr MO, Prosst RL (2016) Design and performance of a low-cost telemetric laparoscopic tactile grasper. Surg Innov 23:291-297

36. Perri MT, Trejos AL, Naish MD, Patel RV, Malthaner RA (2010) New tactile sensing system for minimally invasive surgical tumour localization. Int J Med Robot Comp 6:211-220

37. Reiley CE, Akinbiyi T, Burschka D, Chang DC, Okamura AM, Yuh DD (2008) Effects of visual force feedback on robot-assisted surgical task performance. J Thorac Cardiovasc Surg 135:196-202

38. Haouchine N, Kuang W, Cotin S, Yip M (2018) Vision-based force feedback estimation for robot-assisted surgery using instrument-constrained biomechanical 3D Maps. IEEE Robot Autom Lett 3:2160-2165

39. Schwalb W, Shirinzadeh B, Smith J (2016) A force-sensing surgical tool with a proximally located force/torque sensor. Int J Med Robot 13:e1737

40. Wagner CR, Stylopoulos N, Jackson PG, Howe RD (2007) The benefit of force feedback in surgery: examination of blunt dissection. Presence 16:252-262

Publisher's Note Springer Nature remains neutral with regard to jurisdictional claims in published maps and institutional affiliations. 